tion betrafen, einen an den Abt und Presbyter Elias, und drei an den Presbyter Athanasius. ${ }^{24}$

Die erste Abteilung der $P m b Z$ führt zahlreiche Isaurier, hauptsächlich Bischöfe und Amtsträger in Isaurien für die Jahre 641-867 auf. ${ }^{25}$ Die Liste legt nahe, daß Isaurier nicht verfemt waren, doch weder innerhalb noch außerhalb der Kirchengeschichte noch eine nennenswerte Rolle spielten. Aus dem einfachen Volk sind nur die Angehörigen und Angestellten des Gregorius Decapolita greifbar. Die außerdem noch im Zusammenhang mit Isaurien genannten Amtsträger, die Kommerkiarier, waren höchstwahrscheinlich keine ethnischen Isaurier, da diese Finanzbeamten gewöhnlich nicht aus dem Raum, in dem sie arbeiteten, stammen durften. ${ }^{26}$

\title{
5. Die sogenannte Isaurische Dynastie im 8. Jahrhundert n. Chr.
}

In der mittelbyzantinischen Zeit gibt es eine sogenannte „isaurische Dynastie“ ${ }^{27}$ deren Begründer Leo III. (717-741) den Ikonoklasmus eingeführt haben und aus Isaurien stammen soll. ${ }^{28} \mathrm{Da}$ es jedoch als gesichert gilt, daß Leo III. aus Syrien stammte,

24 Gregor der Große, ep. V 38 p. 762: Heliae, presbytero Isauriae: Excusationem eius accipit; mittit Evangelia; Epiphanium diaconatu Romanae Ecclesiae alligat; modestiam subsidii petitionem liberalitate vincit; ep. VI 66 p. 849: Athanasio, presbytero de Isauria: Ipsum ab omni haereseos labe purum pronuntiat, idque se Constantinopolitano episcopo scripturum spondet; ep. VII 32 p. 889: Athanasio, presbytero de Isauria: De contentione tollenda quae inter patrem monasterii, quod Neas dicitur, et Jerosolymorum episcopum exorta erat.

$25 \mathrm{Zu}$ den Problemen der historischen Repräsentanz der aufgefühtten Personen vgl. PmbZ Prolegomena 285-294. - Für den Hinweis auf die PmbZ danke ich herzlich Herrn Prof. Ralph-Johannes LiLIE.

26 Zur Definition der Kommerkiarier und der byzantinischen Verwaltung Isauriens s.o. III.1.11.

27 Stammtafel in $O D B 2$ (1991) 1015.

28 PmbZ s.n. Leon III. [\# 4242]. Nach Paul SPECK ist Leo III. der Ikonoklasmus (wohl aufgrund seiner rigiden Gesetzgebung) erst zu einem späteren Zeitpunkt angehängt worden. Den Ikonoklasmus habe vielmehr erst sein Sohn Constantin V. (741-775) eingeführt, vgl. SPECK (1998) 58-60: Ursache war ein Streit zwischen Germanus und dem Patriarchen Constantin von Nakoleia. Germanus war für die Bilderverehrung, da er bei der Belagerung von Konstantinopel 717 durch die Araber selbst mit Kreuz und Marienbild auf der Mauer eine Prozession veranstaltet hat, um die Gefahr abzuwenden. Germanus trat auch nicht aus Protest gegen die Bilder-Politik Leos III. zurück, sondern wegen dessen Angliederung der westlichen Kirchenprovinzen (Illyricum, Süditalien und Sizilien) an Konstantinopel. Das Bilderverbot wurde erst durch Constantin V. nach der Pest von 746 erlassen. Die Quellen zu diesem Thema seien mit Vorsicht zu behandeln, da sie nachträglich auf den Bilderstreit hin umgeschrieben wurden. Das Ende des Ikonoklasmus erfolgte 843 durch Kaiserin Theodora, SPECK (1998) 64. Die zeitgenössische muslimische (des Sultans Jazid) und jüdische Bilderfeindlichkeit habe auf Leo keinen Einfluß gehabt: GERo (1973) 59-84. GERo (1974) 
wird diese Dynastie in der neueren Literatur als "syrische Dynastie“ bezeichnet. ${ }^{29}$ Ursache für die Annahme einer ,, isaurischen“ Dynastic im 8. Jahrhundert ist die Behauptung des Theophanes, Leo stamme aus Germanikeia, ,in Wahrheit aus Isaurien" " ${ }^{30}$ sowie die Überlieferung, der Geburtsname des Kaisers habe Konon gelautet, was ein seit jeher bei Isauriern beliebter Name war. ${ }^{31}$ Der Haß auf die Isaurier scheint sich jedoch bis ins byzantinische Frühmittelalter erhalten und auf Kaiser Leo III. gewirkt zu haben. So hätten nach Cedrenus die Griechen die kaiserliche Familie überhaupt ,als ein Bastardgeschlecht von Emporkömmlingen mit einem Kaiser, der selbst ein isaurischer Barbar war" gehaßt. ${ }^{32}$ Darin tritt die von der späteren byzantinischen Literatur getroffene peiorative Bezeichnug der Isaurier zutage: Alle Isaurier waren schlecht, deshalb mußte auch der mutmaßliche Ikonoklast Leo III. aus Isaurien kom-

$38 \mathrm{ff}$. wendet sich auch gegen die These von Peter BRown, 'A Dark-Age Crisis: aspects of the Iconoclastic controversy' in: EHR 346 (1973), 1-34, daß das Motiv für den Ikonoklasmus im zentralistischen Streben des Kaisers gegen die Mönche gelegen habe, denn der eine zentralistische Politik verfolgende Kaiser Heraclius (610-641) habe auf seinem Persienfeldzug Ikonen mitgeführt, und es habe mit Sabbatios auch aus dem Mönchtum stammende Ikonoklasten gegeben.

29 DitTEN (1993) $159 \mathrm{ff}$.

30 Theoph. AM 6209.

31 Parast. 1. 72; GERo (1973) 13-24 zu „Konon“: Untersuchung dieses Namens, Übersicht über die historischen Belege. Er weist nach, daß Konon kein typisch isaurischer Name ist und entkräftet somit das Argument, er müsse aufgrund seines Geburtsnamens ein Isaurier sein. Vermutlich sei ein alter kleinasiatischer oder semitischer Name im Zuge der Hellenisierung des Ostens mit dem alten attischen Namen identifiziert worden (23). Nach SPECK war Konon ein bei Theophanes gebräuchlicher Spitzname für Konstantin gewesen. Zum Namen Konon vgl. auch Rochow (1991) 81. Dafür, daß Leo den Taufnamen Konon als Isaurier getragen habe, vgl. J. KulakovsKIJ, Istorija Vizantii III. Kiew 1915, 319 mit Anm. 2: Leo habe den „für Isaurier typischen“ Taufnamen Konon getragen, (wie es in den Parast. überliefert ist). Doch Ostrogorsky (1963) 129, Anm. 2 führt dagegen an, daß auch Papst Konon (686-687) kein Isaurier, sondern nach dem Lib. Pont. ,natione grecus, oriundus parte [richtig: patre] Tracesio, educatus apud Siciliam“"war. Dazu merkt GERO (1973) 15, Anm. 10-17, Anm. 14 an, daß es eine große Anzahl von Konones aus der klassischen Zeit gegeben habe; vgl. Erich SwOBODA, s.v. 'Konon' RE XI 2 (1922) 1318. Nicht überzeugend V. BENEŠEvIC, 'Sur la date de la mosaique de la Transfiguration au Mont Sinai' in: Byzantion 1 (1924) 159, Anm. 5. - Für die syrische Herkunft sprechen sich noch aus: A. A. VAsiliev, History of the Byzantine Empire, 532-1453, Oxford 21952, 234; Ostrogorsky (1963) 129, Anm. 2; Judith Herrin in: Franz Georg Maler (Hrsg.), Byzanz (= Fischer Weltgeschichte Bd. 13). 1973, 92; weitere Literatur bei RocHow (1991); zuletzt Franz TINNEFELD, s.v. 'Isaurische Kaiser', DNP 5 (1998), 1118: „Dennoch ist der Dynastie bedauerlicherweise - denn es gab ja in der Person von Kaiser Zenon einen wirklichen Isaurier auf dem Kaiserthron - ihr traditioneller Name verblieben“; Robert W. EDWARDs, s.v. 'Isauria', Late Antiquity (1999) $515 f$.

32 Cedr. I p. 796; vgl. Ferdinand Gregorovius, Geschichte der Stadt Athen im Mittelalter, Stuttgart 1889, 108. 
men. ${ }^{33}$ Dies ging so weit, daß sogar ein Kleriker aus jener Zeit, der vom rechten Glauben abgefallen war, im georgischen Synodikon als „Isaurier" bezeichnet wurde. ${ }^{34}$

Die Bezeichnung „Isaurier“ für Leo III. setzte erst spät ein: Von Theophanes abgesehen ist der erste Autor, der Leo einen Isaurier nennt, der nach 843 schreibende Georgius Hamartolus. Hier stellt sich die Frage, wie es dazu kam. Vermutlich liegt eine Verwechslung mit Leos III. Vorgänger Leontius (695-698) vor, der tatsächlich aus Isaurien stammte ${ }^{35}$ und es wegen der unglücklichen Namensgleichheit mit dem Usurpator gegen Zeno wohl vorgezogen hat, sich Leo zu nennen. Dieser Annahme liegt zwar keine historiographische, jedoch eine numismatische Evidenz zugrunde. ${ }^{36}$ Darüber hinaus hat es noch weitere Gründe für die Verwechslung der beiden Kaiser gegeben: beide waren Strategen des anatolischen Themas vor ihrer Thronbesteigung, beide werden von Justinian II. exiliert. ${ }^{37}$

Georgius und die nachfolgenden byzantinischen Chroniken nahmen zumindest die noch bei Theophanes genannte Herkunft Leos aus Germanicia in ihrer Überlieferung aus. Die früheste Quelle, in der Vita des Märtyrers Stephan (gestorben 787), im Jahr 808 verfaßt vom gleichnamigen Diakon der Hagia Sophia, bezeichnet Leo dagegen als Syrer. ${ }^{38}$ Somit kann die Überlieferung, daß Leo III. Isaurier gewesen sei, als eine sekundäre byzantinische angesehen werden. ${ }^{39}$

33 Annahme, „Isaurier“ sei ein „common derogatory epithet“: GERO (1973) 10. Auch nennt Theoph. AM 6232 bei der Beschreibung von Leos letztem Regierungsjahr ihn einen Syrer. Zumindest Maria, Leos Frau, stammte aus Syrien, Parallelquellen zu Theoph. aufgeführt bei RocHOw (1991) 81 .

34 Synodikon der Orthodoxie (BHG 1392) p. 57, 175; PmbZ s.n. 'Paulos' [\# 5855]; möglicherweise aber ein Mißverständnis aus der Wandlung vom „Paulus“ zum „Saulus“ (Eiৎ $\Sigma \alpha \hat{\nu} \lambda o ́ v)$ (ebda.).

35 Franz Tinnefeld, s.v. 'Leontios 9', NP 7 (1999), 66; PmbZ s.n. 'Leontios' [\# 4547]: ,isaurischer, möglicherweise auch armenischer Abstammung".

36 Head (1971) 105-108; vgl. L. LaffranCHI, La numismatica die Leonzio II. Perugia 1940, worin Leontios sich als „Leo" tituliert. Laffranchi hielt dies noch für eine Abkürzung, doch J.P.C. Kent, 'The Mystery of Leontius II' in: Numismatic Chronicle 14 (1954), 217f. hat gezeigt, daß Leontius diesen Namen bei seiner Thronbesteigung übernommen hatte. Auch im Liber Pontificalis und bei Paulus Diaconus, die immer die korrekten Bezeichnungen für die byzantinischen Kaiser benutzen, wird er Leon genannt. Theoph. habe sich zwar erinnert, daß es einen Isaurier auf dem byzantinischen Thron gegeben habe, diesen aber wegen der Namensgleichheit in der Münzprägung verwechselt. Die isaurische Abstammung des Leontius qui et Leo ist durch eine Stelle im Breviarium des Patriarchen Nikephoros belegt, nach welcher Justinian II. während seiner ersten Regierungsphase den zukünftigen Kaiser in seinem Vaterland Isaurien eingekerkert habe: Nikephoros, Breviarium ed. Carl DE Boor, Leipzig 1880, p. 1-77. 37; vgl. K-W 339, Nr. 204.

37 GERO (1973) 75, Anm. 65.

$38 P G$ 100, 1069-1186 (= BHG 1666), 1084 B. 1109 C; vgl. KW 324, Nr. 175.

39 Zum Folgenden vgl. bes. GERo (1973) 3-10; (1974) 24. 
Die orientalischen Quellen (Pseudo-Dionysius von Tell-Mahre, die Kitāb al'Uyūn) bekunden, daß Leon aus dem (zu dieser Zeit schon muslimisch beherrschten) Maraš stammte, welches griechisch Germanikeia hieß. Dies wurde mit dem isaurischen Germanicopolis verwechselt. Schon SCHENK (1896) $296 \mathrm{ff}$. hatte aufgezeigt, $\mathrm{da} \beta$ das isaurische Epitheton aus Theophanes eine Fehlbezeichnung war. Laut SCHENK hatte Theophanes einfach das in der syrischen Commagene gelegene Germanicia mit dem isaurischen Germanikeia verwechselt. Der Widerspruch sei durch einen

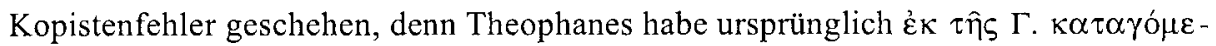

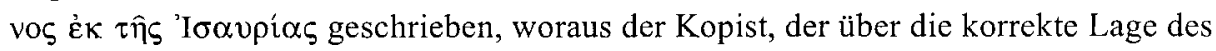
syrischen Germanicia Bescheid wußte, geschrieben habe, daß „in Wahrheit" Isaurien gemeint sei, was die Konfusion nur noch verschlimmerte. In der zeitgenössischen Literatur, bei Johannes von Damaskus und in den römischen Aktenstücken finde sich dieser Beiname kein einziges Mal. Auch habe sich Leo selbst niemals als Isaurier bezeichnet. Ebensowenig wisse Nikephoros Patriarches, der um dieselbe Zeit wie Theoph. schrieb, etwas von einer isaurischen Herkunft des Leo. Karl SCHENK folgerte daraus, daß deshalb auch in der gemeinsamen Vorlage von Theoph. und Nikephoros nichts von einem Isaurier gestanden haben könne. Die Stelle ,in Wahrheit aus Isaurien" sei eine später eingefügte Glosse aufgrund der Tatsache, daß auch die dem päpstlichen Bibliothekar Anastasius zugeschriebene Übersetzung des Theophanes ins Lateinische aus dem 9. Jh. ${ }^{40}$ Isaurien nicht erwähnt, sondern vielmehr davon spricht, daß Leo genere Syrus gewesen sei. Auch seien seine Arabischkenntnisse bezeugt, ${ }^{41}$ was eher für eine syrische denn eine isaurische Herkunft spricht. Anders Averil $\mathrm{CA}_{\mathrm{A}}$ MERON und Judith HERRIN: ${ }^{42}$ Sie sprechen sich aufgrund ihrer frühen Datierung der Parast. als zeitgenössisch, da sie zum Großteil vor den ikonoklastischen Maßnahmen Leos im Jahr 726 verfaßt seien, und ihrer inhaltlichen Verbindung mit der Regierungszeit Leos III. gegen die Thesen von Constance HEAD (1971) und Stephen GERO (1973) aus. HEAD ignoriere die Tatsache, daß Leo III. bereits in den Parast. 1 und 72 „Konon der Isaurier“ genannt wurde. Sie schließen auch die These von „Isaurier“ als einem Schimpfwort und einer Interpolation in die Parast. aus, da sie an dieser Stelle glaubwürdig seien und schlagen vor, daß er von isaurischer Abstammung gewesen sein müsse. Theoph. AM 6221 überliefert, daß Leo im Gespräch mit dem Patriarchen Germanos gesagt habe, sein Geburtsname sei Konon; ebenso in Parast. 20, 1; Adversus Constantinum Caballinum (zwischen 775 und 787 verfaßt) $P G$ 95, $336 \mathrm{C}$ - hiernach soll er diesen Namen auf Anraten eines Juden, der ihm die Herrschaft prophe-

40 Chronographia tripertita, ed. Carl DE BOOR, 251.

41 Ernest W. Brooks, 'The Campaign of 716-718 from Arabic Sources' in: JHS 19 (1899), $21 \mathrm{f}$.

42 Parast. (1984) 168. 17. 21, gefolgt von MANGo / SCotT (1997) 547, Anm. 1 (Kommentar zu Theoph. AM 6209) und Elton (2000) 397, Anm. 22. 
zeihte, abgelegt haben - und späteren Quellen. ${ }^{43}$ Judith HERRIN hatte sich noch gegen die isaurische Herkunft ausgesprochen. ${ }^{44}$ Doch Stephen Gero hatte auf das bereits zu einem sehr frühen Zeitpunkt erfolgte Verdrängen der ,syrischen“ Herkunft durch die ,isaurische" hingewiesen. ${ }^{45}$ Für die isaurische Herkunft spricht sich noch Lucian LAMZA aus; ${ }^{46}$ er versucht eine Harmonisierung der Quellen und schließt sich der These von Cyril MANGO an, wonach Konon qui et Leo III. zu den isaurischen „displaced persons" gehört haben muß, die in der Folge des Krieges gegen die Isaurier unter Anastasius ihre Heimat verlassen hatten. ${ }^{47}$ Der Hinweis, daß Leo, wenngleich aus Syrien gebürtig, dennoch ein Isaurier war, sei so durch seine Abstammung motiviert worden. Dies zeige sich zumindest in der Erwähnung, daß man sein Isauriertum als Hinweis auf seine bilderfeindliche Politik gesehen hat. Weniger überzeugend erscheint die Replik auf GERO von G. L. HUXLEY mit der These, Leo sei sowohl Syrer als auch Isaurier gewesen. ${ }^{48}$ Als Constantin V. Germanicia wiedererobert hatte, habe er die Verwandten seiner Mutter nach Konstantinopel gebracht. HuXLEY schließt daraus, daß Leo keine Verwandten dort besessen haben müsse. Anastasius könnte in seiner Übersetzung einen griechischen Text vorliegen gehabt haben, in welchem das

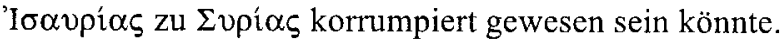

Auch die These von Gero, die Deportation Leos III. mit seinen Eltern nach Thrakien sei eine Erfindung ex eventu des Theophanes, ist nicht unumstritten. ${ }^{49}$

43 Rochow (1991) 123; Mango/ Scott (1997), Theoph.-Kommentar, 565, Anm. 2.

44 In: Franz Georg MaIER (Hrsg.), Byzanz (= Fischer Weltgeschichte Bd. 13). 1973, 92.

45 Gero (1973) 76, Anm. 71. Auch KW 321, Nr. 170 schließen eine spätere Entstehungszeit der Parast. nicht aus.

46 LAMZa (1975) 117f. mit Anm. 12. (126f.).

47 MANGo (1966) 363.

48 G. L. HuXLEY, 'Hagiography and the first Byzantine iconoclasm' in: Proceedings of the Royal Irish Academy 80 C. Dublin 1980, 187-196, 188, Anm. 8.

49 Theoph. AM 6209; Gero (1973) 11, Anm. 43, 30 mit Anm. 20. Dagegen wendet sich Lilie (1976) 235f. mit Anm. 126, 237, Anm. 131, 241 mit Anm. 150, 248 mit Anm. 181, 251f.; nach DitTEN (1993) 161 sei dies geschehen, um die christliche Bevölkerung Thrakiens zu stärken und vielleicht nach einem Vertrag mit den Arabern eine Pufferzone zu schaffen. 